\title{
Discrimination of melodies from the first and fifth serials of the pentatonic scale
}

\author{
W. A. WILBANKS and M. W. PATE \\ University of Georgia, Athens, Georgia 30602
}

\begin{abstract}
A basic aspect of music is the scale or set of tones that is used to generate melodic and rhythmic patterns. There is one scale that has a prominent place in the music of many cultures. This scale, the pentatonic, dates back as far as 1300 B.C. and extends to the present. Data are presented on the discrimination of melodies randomly generated from the first and fifth serials of the pentatonic scale. The question is: does the part of the scale used to generate a melody give a distinct quality to the melody? The results show that such melodies can be discriminated independently of melodic line, rhythm, frequency range, and tone color. This suggests that melodies can be heard as being from two different serials, indicating that each of these serials possesses a quality, a tonality, that yields perceptually different melodies. The results are related to Orff's theory of musical development.
\end{abstract}

A basic aspect of music is the scale or set of tones that is used to generate melodic and rhythmic patterns. In every time and place, there is found a musical culture that bases its performances on an organized framework of tones whose fundamental frequencies have systematic relations to each other. For these systems, diatonic, pentatonic, or otherwise, the general term "tonality" is employed. Although the range of pitches can be treated as a continuum, musical practice does not treat it as such. Instead, the pitch range is divided up into a scale on which the notes of musical performance can be located.

There is one scale that has a prominent place in the music of many cultures. This scale, the pentatonic scale, dates back as far as 1300 B.C. and extends to the present. Kuttner (1964) reports evidence that the pentatonic scale was used in China as early as the Shang II dynasty. Sets of stones have been found that were tuned to the notes of the pentatonic scale. Kodály (1970) has performed extensive analyses of Hungarian folk music and found a widespread usage of the pentatonic scale. Ornstein (1971) also reports the use of the pentatonic scale in Bali folk music. According to Sharp (1965), the pentatonic scale is common in Scotland, particularly in tunes of Gaelic origin. He also states that a large proportion of tunes collected from the southern Appalachian mountains in America are pentatonic. Pentatonic melodies can also be found in hymn books used by many Christian denominations present in the southern states of America. The Broadmal Hymnal and the Methodist Hymnal contain many songs that employ melodies derived from the pentatonic scale. Not only are these pentatonic-based hymns present today, but they were present in a hymn book that was the widespread source of American church music in the 19th century, The Original Sacred Harp (published in 1844). Besides the hymn book sources of pentatonic songs, there is a secular source known as The Social Harp, originally published in 1855. Most of the pentatonic songs can be traced back to European origins (Sharp, 1965). Burton (1901) demonstrates that many American Indian songs are based upon the pentatonic scale. Folk songs from China, Japan, and Korea show an exclusive use of the pentatonic scale.

In addition to its antiquity and ubiquity, the pentatonic scale has some most interesting features from the point of view of the psychology of music. It was in 1772 that the French composer Rameau (1772/1971) formalized the modern system of tonality in his Génération Harmonique. The main aspect of Rameau's system was not the traditional system based upon the church modes, but one based upon groups of three tones called triads. Rameau's theory was based upon two kinds of triads, major and minor. The tonality of tones sounded sequentially as well as concurrently could be analyzed by determining how the tones fit different patterns generated by the two basic kinds of triads. A major triad and a minor triad can be thought of as two separate scales that have three tones each. The ratios of the fundamental frequencies of the first and third tones are the same for both triads. The second tone is the only distinction between the two triads, that is, a major third or a minor third. Besides Rameau's theorizing and the importance ascribed to the majorminor distinction in harmony, there is a body of experimental evidence that the major-minor polarities do exist in our perception of music. Hevener (1935) presented different melodies first in a major mode and then in a minor mode. Her subjects consistently rated the two different modes according to the categories related to "bright" and "happy" for the major mode, and "sad" and "dark" for the minor mode. Corso (1957) found that subjects could accurately distinguish on the basis of absolute judgments between major and 
minor chords. An interesting feature of the pentatonic scale is that the major-minor polarity applies, in that the first serial of the pentatonic scale contains a major triad in root position, while the fifth serial contains a minor triad in root position. The first and fifth serials are the most distinct from the point of view of Rameau's treatment of tonality. The ethnomusicology literature also indicates the widespread use of these two serials of the pentatonic scale in musical practice.

The first and fifth serials are composed of the following intervals, formed by comparing each tone in the serial with the lowest tone in the serial (first) 1 , $2 \mathrm{M}, 3 \mathrm{M}, 5 \mathrm{th}, 6 \mathrm{th}$; (fifth) $1,3 \mathrm{~m}, 4 \mathrm{th}, 5 \mathrm{th}, 7 \mathrm{~m}$. The interval between any adjacent tone in the equally tempered scale is a minor second. As the interval is expanded by successive tones, the interval becomes a $2 \mathrm{M}, 3 \mathrm{~m}, 3 \mathrm{M}$, and so on. There are only two different intervals between adjacent tones in the first and fifth serials. These are a major second and a minor third. The only distinction between parallel serials of the pentatonic scale is the arrangement of these adjacent intervals: (first) $2 \mathrm{M}, 3 \mathrm{~m}, 2 \mathrm{M}, 2 \mathrm{M}, 3 \mathrm{~m}$; (fifth) $3 \mathrm{~m}, 2 \mathrm{M}$, $3 \mathrm{~m}, 2 \mathrm{M}, 2 \mathrm{M}$. Jeffries (1967) has shown that these are two of the easiest intervals to recognize. It is also important to note that the dissonant interval of the minor second is not present. This is perhaps the clearest distinction between the pentatonic scale and the diatonic and 12-tone chromatic scales. As the number of tones in a scale increases, the number of possible dissonant intervals increases. There is a tradeoff between consonance and variety in scale construction. The pentatonic scale has been termed a "gapped" scale because the minor second is omitted as an adjacent interval. The work of Plomp and Levelt (1962) suggests that the relative consonance of two tones varies as a function of the fraction of a critical bandwidth separating their frequencies. Although, as Ward (1970) emphasizes, it is not clear whether Plomp and Levelt's listeners used as their criterion of consonance what "everyone uses (especially musicians)," their results are consistent with the remarkable feature of the pentatonic scale that all the pentatonic tones can be played as a harmonious accompaniment to any pentatonic melody.

An additional feature of psychological interest needs to be emphasized, the use of the pentatonic scale in systems of music education. Two widely discussed music educators and distinguished composers, Carl Orff and Zoltan Kodály, have proposed theories that there are discrete stages in the development of musical competence. Both Orff and Kodály have suggested that musical development procedes through discrete stages defined by the complexity of the scale used to generate musical patterns. The Orff formula for tonal learning begins with a two-tone pattern, a descending minor third, Tones 5 and 3 . A third tone is added for the next stage, Tone 6 . In the third stage, Tones 2 and 1 are added, giving the pentatonic scale with its harmonic involvement (Flagg, 1966; Landis \& Carder, 1972; Nash, 1968). The Orff-Schulwerk (Orff \& Keetman, 1956) is a collection of music that starts with the pentatonic scale and then proceeds to major and minor diatonic scales based upon the major and minor triads.

To summarize the preceding discussion, (1) the pentatonic scale is widely used as a source of music throughout the world; (2) the first and fifth serials are related to theories of tonality that emphasize a majorminor tonality; (3) the intervals present in the scale are ranked high on tests that measure consonance and recognition; and (4) the pentatonic scale has a prominent position in the theories of musical development of Orff and Kodály.

Now, it has often been maintained that the various scales possess certain perceptible characteristics that make them appear qualitatively different. Thus, for example, each key of the major and minor modes of the equally tempered scale has been said to have, to quote from Révész (1954), an "inherent expressive value peculiar to that key," such that a listener can recognize the key or tonality of a given composition by its particular "color" or "mood." In a definitive study, Corso (1957) found that, although college musicians can identify certain keys or serials of the equally tempered scale on the basis of absolute judgments, his results failed to support the classical theory that different scales possess inherent qualities that permit the listener to distinguish among them solely on the basis of tonality. Now, the scale is a product of melody. Although readers of Helmholtz (1954) would be tempted to identify theoretical order with genetic order, it is clear today that we have scales as a consequence of having made certain melodies, and not that we make melodies as a consequence of having scales. We can clearly distinguish between melodies on the basis of melodic line-the pattern of successive tones of different frequency content-rhythm, "tone color," pitch range, and the like. Although we may not be able to distinguish between scales on the basis of tonality, it does not necessarily follow that we cannot distinguish between melodies on the basis of the particular part of the scale on which the notes can be located, independently of melodic line, rhythm, pitch range, and so on. The question here is: do melodies generated from different serials of a scale possess perceptible qualities that permit the listener to distinguish between them on the basis of tonality, that is, the particular part of the scale on which the notes of musical performance can be located?

Now, hearing a 12-tone series is not the same thing as hearing it as a 12-tone series, that is, naming it and giving it a structure. Likewise, hearing two melodies generated from different serials of a scale is not the same thing as hearing them as from two different serials. The present study focused on this question: Can we 
discriminate between melodies randomly generated from different serials of the pentatonic scale independently of melodic line, rhythm, tone color, and pitch range? In other words, can we discriminate between melodies generated from two serials of the pentatonic scale as being from two different serials?

\section{METHOD}

The stimuli consisted of pairs of melodies generated by randomly selecting tones from the first and fifth serials of the pentatonic scale covering the same one-octave range (parallel serials). Each melody in a pair contained 20 sinusoidal signals. On one-half of the trials, the melodies were from different serials (presented in $\mathrm{AB}$ or $\mathrm{BA}$ order); on the other half of the trials, bcth melodies were obtained by random selection of tones from the same serial (AA or BB). Each tone was presented with an on-off time of $20 \mathrm{msec}$. The signals used to generate melodies from the first serial were $349,392,440,523,587$, and $699 \mathrm{~Hz}$ (in musical notation, F, G, A, C, D, F'). The signals for the parallel fifth serial were $349,415,466,544,622$, and $699 \mathrm{~Hz}$ (F, A flat, B flat, D flat, E flat, and $F^{\prime}$ ). Each block of trials consisted of 54 melodic pairs.

The time between the onset of each tone (meter length) was constant for a given pair of melodies, but was varied in the different pairs from 350 , to 550 , and to $750 \mathrm{msec}$. The duration of each tone in a given pair was the same, but was varied across melodic pairs according to the values of $17 \%, 39 \%$, and $61 \%$ of the meter length. In this manner, both rhythm and attack characteristic (staccato-legato) were parametrically varied. The melodic line (pattern of successive tones of different frequency) of each melody was determined by random selection from the two serials.

Between each melodic line in a pair, there was a 2 -sec period of silence. Between each pair, there was a 5-sec period of silence to permit the listener to make a response. Eighteen melodic pairs were presented initially, then a 3-min rest was given, followed by 18 more melodic pairs, a $3-\mathrm{min}$ rest period, and, finally, 18 more melodic pairs to complete a block of 54 trials. A complete block of trials lasted approximately $30 \mathrm{~min}$. The order of melodic pairs in a block was random with respect to the different parameter values. The complete block of 54 melodic pairs was recorded and reproduced on magnetic tape and was presented on each of 8 successive days. Each listener heard a total of 432 melodic pairs. The sound patterns were presented via TDH-49 earphones at a level of $80 \mathrm{~dB}$ SPL $( \pm 1.5 \mathrm{~dB})$. Listening was done in a standard IAC booth.

The listener was provided with two response keys and instructed to press one key if the two melodies in a pair were judged to be from the same serial of the pentatonic scale, and to press the other key if the melodies were from different serials. The listener was instructed to make a response even when uncertain of the judgment.

Two groups of subjects were used. One consisted of 16 college musicians at the University of Georgia. The other consisted of 40 nonmusic students; 33 subjects were obtained from sections of introductory psychology, and 7 were graduate students in psychology.

\section{RESULTS AND DISCUSSION}

The mean percent correct judgments for music and nonmusic subjects were approximately $80 \%$ and $71 \%$, respectively. Both of these performance levels are significantly better than chance (ts significant beyond the .001 level). The difference between music and nonmusic students was significant beyond the .01 level $(\mathrm{t}=2.93)$

The three different conditions of tone duration and meter length were combined and organized into a 3 by 3 factorial design for each group (equal $n$, repeated measures). The results of these analyses show that the differences in performance due to varying tone duration and meter length were not significant at the .05 level. There was no significant interaction between meter length and tone duration.

These results indicate that listeners can discriminate between melodies generated from the first and fifth serials of the pentatonic scale independently of melodic line, tempo, and tone color (attack characteristics). In short, melodies from the first and fifth serials can be heard as being from two different serials, indicating that each of these serials possesses a quality, a tonality, that yields perceptually different melodies.

The relative difficulty of nonmusic students in discriminating the serials relative to music students may be related to Orff's theory of musical development. There are two ways that serials of the pentatonic scale may be generated. One way is to generate the scale over two octaves and compare the different serials as they exist in the different segments of the two-octave span. This may be termed a linear comparison. Such a comparison was not used in the present study because the difference in frequency of the lowest and highest tones in each serial would be an obvious basis for making the discrimination. Instead of this linear comparison, a parallel comparison was made. Here, the tones in the two serials were adjusted so that the lowest and highest tones in each serial had the same frequency, while the ratios of the tones in each serial was retained. In this way, the two serials had the same frequency range. A comparison of this nature, however, requires the use of a scale more complex than the five-tone scale. When the tones in both serials are combined in a parallel arrangement, the result is a scale that has a total of eight different tones within a given octave. According to Orff's theory, the number of tones used in a scale is related to the difficulty of generating and recognizing different forms of music within the given scale. Since an eight-tone scale is needed to generate two parallel serials, it might be argued that recognizing the different parallel serials of the pentatonic scale is a perceptual task more difficult than recognizing forms generated exclusively from the linear forms. That is, the recognition of parallel serials of the five-tone pentatonic scale is an intermediate step in developing the ability to recognize musical forms related to a more complicated scale, such as the diatonic. The listeners who found the task of discriminating the two parallel serials relatively easy may represent a category of listeners who have already mastered the diatonic scale. The listeners who found the discrimination relatively difficult may represent a category of listeners who had not completely 
mastered the diatonic scale. The wide range of values of percent errors for the different listeners, as well as the continuous nature of the distribution, also suggests that mastery of the pentatonic and diatonic scales is not an all-or-none process, but one that develops by gradual steps. The use of different parallel serials of the pentatonic scale may be a means of introducing the diatonic.

In summary, melodies randomly generated from the first and fifth serials of the pentatonic scale can be discriminated independently of melodic line, rhythm, frequency range, and tone color. The data suggest that mastery of the pentatonic scale is not abrupt, but one that has several stages of development. Both parameters of tone duration and meter length had negligible effects upon the serial discriminations. This suggests that the pentatonic scale is a stable source for making soundpattern discriminations and is relatively unaffected by the manipulation of other parameters of the sound patterns.

\section{REFERENCES}

Burton, F. R. American primitive music. New York: Moffat, Yard, \& Company, 1901.

Corso, J. F. Absolute judgments of musical tonality. The Journal of the Acoustical Society of America, 1957, 29, 138-144.

FLAGG, M. The Orff system in today's world. Music Educators Journal, December 1966, 30.

HeLmHOLTZ, H. L. F. [On the sensations of tone as a physiological basis for the theory of music] (Ellis, trans.), New York: Dover, 1954.
HEVENER, K. The affective character of the major and minor modes in music. The American Journal of Psychology, 1935, 47, 103-119.

JEFFRIES, T. B. The effects of order of presentation and knowledge of results on the aural recognition of melodic intervals. Journal of Research in Music Education, 1967, 15, 179-190.

KodÁly, Z. Pentatonicism in Hungarian folk music. Ethnomusicology, 1970, 14, 228-241.

KuTtNer, F. A. The music of China: A short historical synopsis incorporating the results of recent musicological investigations. Ethnomusicology, 1964, 8, 121-127.

LANDIs, B., \& CARDER, P. The eclectic curriculum in American music education: Contributions of Dalcroze, Kodály, and Orff. Washington, D.C: Music Educators Conference, 1972.

Nash, G. Kodály and Orff. Clavier, September 1968, 18-19.

OrfF, C., \& KeEtman, G. Orff-Schulwerk (Vol. I). New York: Schott, 1956.

OrNstein, R. The five-tone Gamelan Angklung on North Bali. Ethnomusicology, 1971, 15, 71-80.

Plomp, R., \& Levelt, W. J. M. Tonal consonance and critical bandwidth. The Journal of the Acoustical Society of America, 1962, 38, 548-560.

RAmEAU, J. P. Génération harmonique [Treatise on Harmony] (P. Gossett, trans.). New York: Dover, 1971. (Originally published, 1772.)

REVÉSZ, G. Introduction to the psychology of music. Norman, Okla: University of Oklahoma Press, 1954.

Sharp, C. J. English folk songs. San Francisco: Wadsworth, 1965.

WARD, W. D. Musical perception. In J. V. Tobias (Ed.), Foundations of modern auditory theory (Vol. I). New York: Academic Press, 1970.

(Received for publication November 3, 1978.) 